\title{
Inter-Comparison of SMOS and Aquarius Sea Surface Salinity: Effects of the Dielectric Constant and Vicarious Calibration
}

\author{
Emmanuel P. Dinnat \\ Chapman University \\ NASA-Goddard Space Flight Center \\ Greenbelt, MD, U.S.A. \\ emmanuel.dinnat@nasa.gov
}

\author{
Jacqueline Boutin and Xiaobin Yin \\ LOCEAN, CNRS/IRD/UPMC/MN \\ Paris, France
}

\author{
David M. Le Vine \\ NASA-Goddard Space Flight Center \\ Greenbelt, MD, U.S.A.
}

\begin{abstract}
Two spaceborne instruments share the scientific objective of mapping the global Sea Surface Salinity (SSS). ESA's Soil Moisture and Ocean Salinity (SMOS) and NASA's Aquarius use L-band $(1.4 \mathrm{GHz})$ radiometry to retrieve SSS. We find that SSS retrieved by SMOS is generally lower than SSS retrieved by Aquarius, except for very cold waters where SMOS SSS is higher overall. The spatial distribution of the differences in SSS is similar to the distribution of sea surface temperature. There are several differences in the retrieval algorithm that could explain the observed SSS differences. We assess the impact of the dielectric constant model and the ancillary sea surface salinity used by both missions for calibrating the radiometers and retrieving SSS. The differences in dielectric constant model produce differences in SSS of the order of 0.3 psu and exhibit a dependence on latitude and temperature. We use comparisons with the Argo in situ data to assess the performances of the model in various regions of the globe. Finally, the differences in the ancillary sea surface salinity products used to perform the vicarious calibration of both instruments are relatively small $(0.1$ psu), but not negligible considering the requirements for spaceborne remote sensing of SSS.
\end{abstract}

Keywords— sea surface salinity; L-band; radiometry; calibration; Aquarius; SMOS; dielectric constant

\section{INTRODUCTION}

Both ESA's Soil Moisture and Ocean Salinity (SMOS) [1] and NASA/CONAE's Aquarius/SAC-D [2] missions share the common objective to monitor global Sea Surface Salinity (SSS). Both missions aim at producing monthly global SSS maps with a precision of the order 0.2 practical salinity unit (psu) at a spatial resolution of the order of $150 \mathrm{~km}$. They share common features: e.g. use of L-band $(1.41 \mathrm{GHz})$ radiometry as prime instrument for measuring SSS and sun-synchronous polar orbits. They also have some significant differences in their approaches. SMOS uses interferometry to produce images, while Aquarius uses three beams with fixed pointing across the orbital plane toward the night side of the Earth. As a result, SMOS resolution $(\sim 40 \mathrm{~km})$ is higher than Aquarius' $(\sim 100 \mathrm{~km}-150 \mathrm{~km})$, each location on Earth is seen by SMOS under a wide range of incidence angles as the spacecraft moves forward. On the other hand, Aquarius has a few advantages over SMOS: its radiometric sensitivity is much better $(\sim 0.12 \mathrm{~K}$ over $1.44 \mathrm{sec}$. integration time), it has advanced Radio Frequency Interferences (RFI) filtering by using very short time integration subsamples (of the order of $10 \mathrm{~ms}$ ) and it uses a scatterometer to help with the correction of sea surface roughness effects. In addition, its beams point towards the night side of the Earth to avoid Sun contamination [3]

Both missions also have similarities and differences in the algorithm used to retrieve SSS. They both apply a post launch vicarious calibration to the radiometric measurements, derived by comparing the measurements to a forward radiative transfer model. However, the models and the locations on the globe and time period used for performing the calibration differ notably. It should be noted that in the case of SMOS, only the level 2 data over ocean, used for SSS retrieval, include the vicarious calibration discussed here. Level 1 data and data over land and ice do not include this processing.

Significant differences in SSS retrieved by both sensors are observed. SMOS SSS is generally lower than Aquarius SSS, except for the coldest waters in the high southern latitudes. Fig. 1 (top) is an example of the differences averaged over one month and in $1^{\circ}$ bins in longitude and latitude. Differences are mostly between -1 and +1 psu, with a significant regional, latitudinal (Fig. 1, middle) and temperature dependence (Fig. 1, bottom). Large differences around coasts are likely due to land contamination in the SMOS signal. We investigate the impact of the vicarious calibration and retrieval algorithm on the noncoastal differences. In Section II, we present the differences in algorithms regarding the dielectric constant model and the ancillary SSS product used for the calibration. In Section III, we report the impact of the dielectric constant model on the SSS retrieved by Aquarius and compare with SMOS. In Section IV, we compare Aquarius SSS obtained with different dielectric constant models to in situ data. In Section V, we

This research was supported by the NASA grant \# NNX12AG69G. 

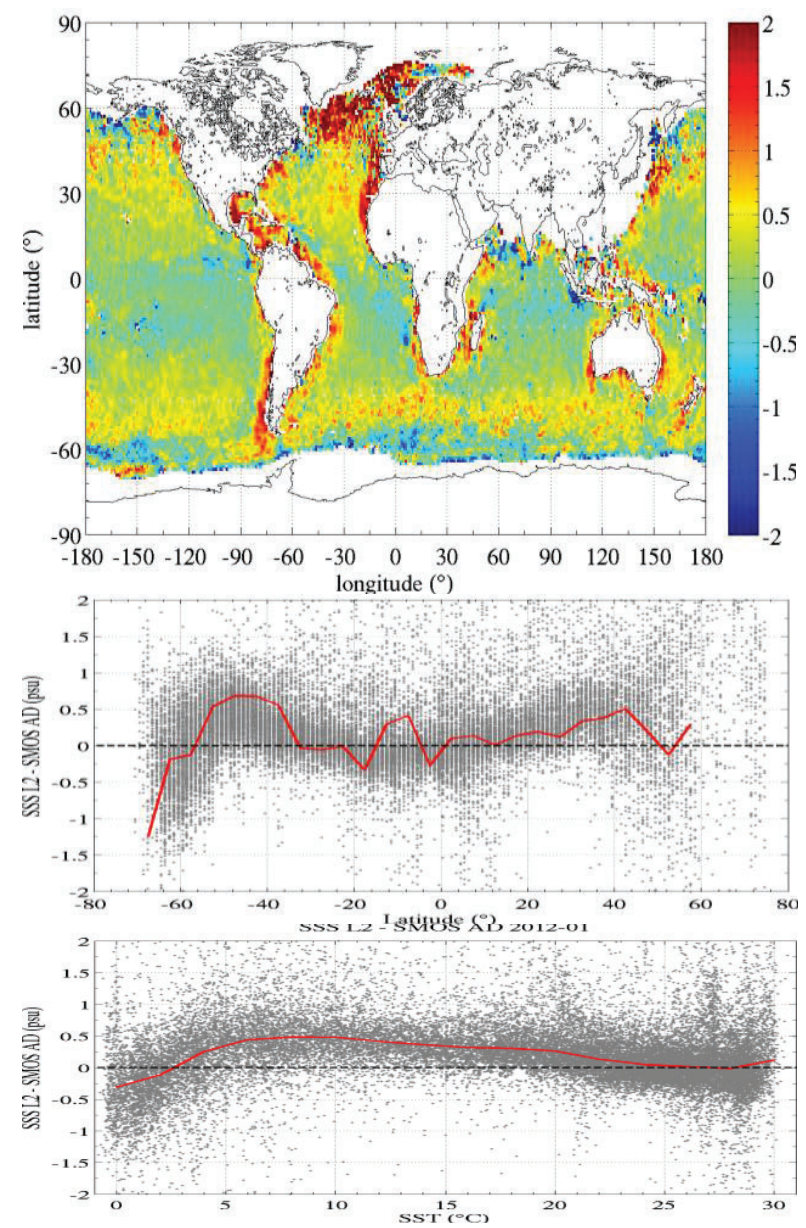

Fig. 1. (top) Global map of the differences in SSS (psu) Aquarius minus SMOS for the month of Jan 2012. The same data as in (top) are reported versus (middle) latitude and (bottom) sea surface temperature. The red line reports the median of the difference inside (middle) $5^{\circ}$ bins in latitude and (bottom) $2^{\circ} \mathrm{C}$ bins in SST.

discuss the differences between the ancillary SSS used for calibration. In Section VI, we summarize the results and discuss their implication and future research.

\section{DIFFERENCES IN SMOS AND AQUARIUS ALGORITHMS}

Radiometric measurements from SMOS and Aquarius are calibrated post-launch using comparisons with a forward radiative transfer model. Aquarius forward model is described in [4]. There are notable differences in the way this calibration is performed by both missions. First the forward models use different parameterizations for the dielectric constant and the sea surface roughness. Second, the ancillary data (SST, SSS, wind) used as input to the forward model come from different sources. Finally, the domain (space, time, reference frame) where the calibration is performed differ. For SMOS, the comparison concerns brightness temperatures in the antenna reference frame over a small region of the Pacific Ocean [5]. In the case of Aquarius, the comparison concerns antenna temperatures (i.e. including the effects of the atmosphere, the Faraday rotation, antenna pattern and celestial sources) and are performed globally. In this study, we assess the impact of two
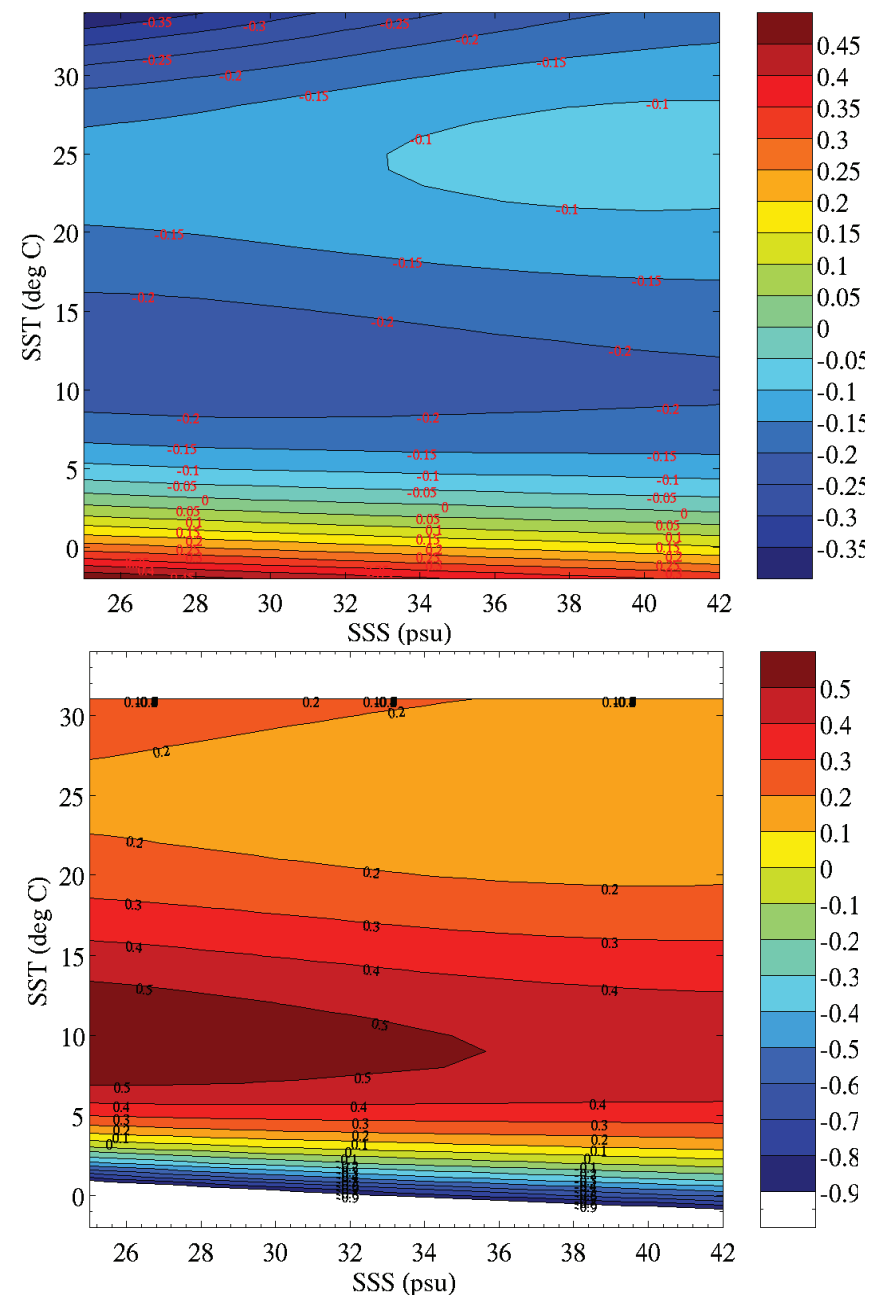

Fig. 2. Effect of differences in dielectric constant (KS minus MW) on (top) $\mathrm{Tb}$ at vertical polarization and (bottom) retrieved SSS.

aspects of the calibration: the dielectric constant model and the ancillary SSS product used for the forward model.

\section{A. Differences in dielectric constant model}

The dielectric constant of sea water has been shown to be uncertain at L-band [6][7]. The differences between existing models lead to differences in Tb of as much as a Kelvin. The differences vary with SST, and SSS in cold waters. Such differences are significant for retrieving SSS with a precision of the order of $0.2 \mathrm{psu}$ [7]. An error in $\mathrm{Tb}$ of $0.1 \mathrm{~K}$ translates into an error of $0.2 \mathrm{psu}$, or more in cold waters.

SMOS uses the dielectric constant model by Klein and Swift (KS) [8]. Aquarius uses a more recent model developed by Meissner and Wentz from radiometric measurements [9]. We report the difference in $\mathrm{Tb}$ for a flat surface (i.e. Fresnel coefficients, here at an incidence angle of $38^{\circ}$ ) computed using both models in Fig. 2 (top). The horizontal stratification of the contours indicates that the difference is mostly sensitive to SST and much less sensitive to SSS. Over most oceans (i.e. for SST greater than $4^{\circ} \mathrm{C}$ ), the $\mathrm{KS}$ model predicts a Tb smaller by a few tenths of a Kelvin than the MW model. For the coldest waters (less than $10^{\circ} \mathrm{C}$ ), the difference becomes very sensitive to SST. 


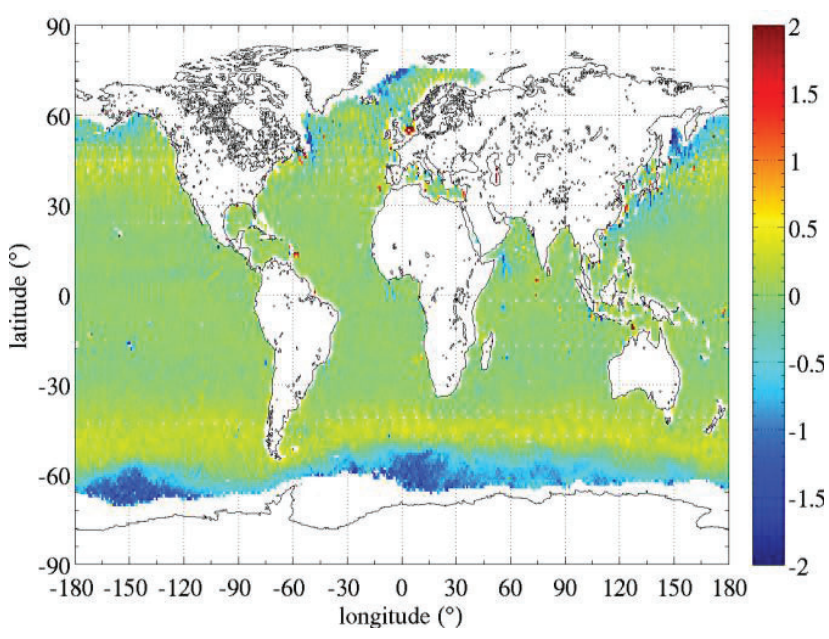

Fig. 3. Global map of the differences in SSS (psu) between the nominal Aquarius Level 2 product and Aquarius data reprocessed using the KS dielectric constant model for the month of Jan 2012..

The KS model predicts larger Tb for very cold waters (SST less than $3^{\circ} \mathrm{C}$ ). We compute an error in SSS due to the differences in $\mathrm{Tb}$ by computing a "true" Tb using the KS model and inverting to determine SSS using the MW model. The SSS error is mostly positive (i.e. for SST warmer than $4^{\circ} \mathrm{C}$ ), and up to $+0.5 \mathrm{psu}$ for SST around $10^{\circ} \mathrm{C}$ (Fig. 2, bottom). For the very cold waters, the SSS error becomes negative (up to $-1 \mathrm{psu}$ ), and changes rapidly with SST.

\section{B. Ancillary Sea Surface Salinity for Vicarious Calibration}

There are differences in the way SMOS and Aquarius perform the vicarious calibration over the oceans. SMOS so called Ocean Target Transformation (OTT) uses comparisons between measured Tb's and forward model simulations over a limited region in the Pacific Ocean to remove biases in its field of view [5]. Aquarius performs a comparison on the antenna temperatures at global scale [10]. The reference SSS for the forward model simulations is the World Ocean Atlas (2009) for SMOS [11] and the HYbrid Coordinates Ocean Model (HYCOM) for Aquarius [12]. We compute monthly averages of the HYCOM maps to have an equivalent to the WOA climatology maps in order to assess the difference in the reference salinity fields that are used to calibrate both instruments.

\section{IMPACT OF THE DieleCtRIC CONSTANT MODEL ON AQUARIUS SSS}

The dielectric constant model is used at two stages of the data processing. First, it is used in the calibration of the instruments, by comparing radiometric measurements to forward model simulations. Second, it is used to invert SSS from surface brightness temperature. In order to assess the impact of the dielectric constant on the differences observed in SSS between SMOS and Aquarius, we reprocess the Aquarius data using the KS model used for SMOS. We use the KS model for the reference ocean used in the calibration of Aquarius; then we used it again, keeping all other factors the same, to perform the inversion to obtain SSS.
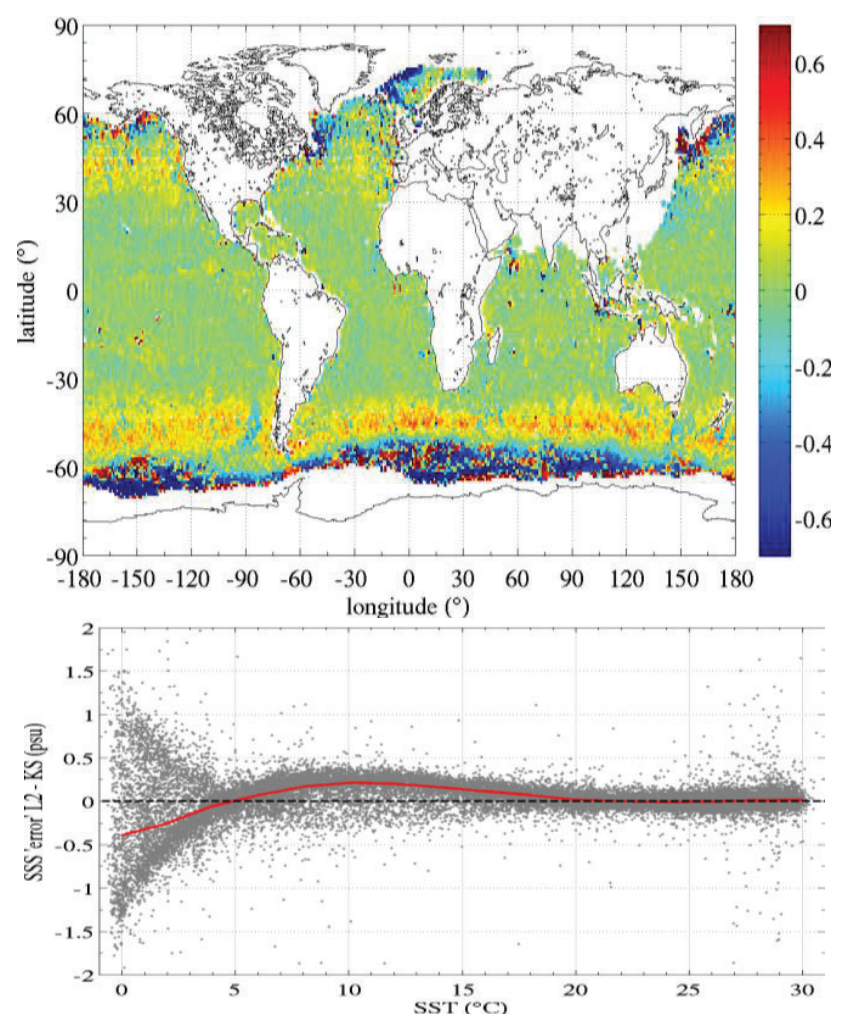

Fig. 4. (top) Map of the impact of using the KS dielectric constant model on the monthly (Jan 2012) difference Aquarius minus SMOS. (bottom) Same data as in the map at the top reported versus SST. The red line reports the median of the difference inside $2{ }^{\circ} \mathrm{C}$ bins in SST.

Differences in SSS between Aquarius nominal level 2 data and data derived after our reprocessing using the KS model is reported in Fig. 3. The differences vary mostly within 0.5 psu at global scale, with a few larger regional variations at high latitudes and in cold waters. Seasonal variations occur at mid and high latitudes (not shown). One can observe that at low and mid latitudes, namely for temperate and warmer waters, differences are very small. This is a different result than reported in Fig. 2 (bottom). This is because we have calibrated the Aquarius data with the KS model before performing the inversion to SSS, de facto removing a global average bias. The differences exhibit large scale patterns similar to those in Fig. 1. There are two latitude bands around $-45^{\circ}$ and $+45^{\circ}$ where the SSS difference is positive (light yellow). In the very high southern latitudes $\left(-60^{\circ}\right)$, the difference is reversed and becomes negative, around -1 psu (blue).

The large scale patterns of the difference in Fig. 3 follow the large scale patterns of SST (not shown), similarly to the differences in Fig. 1. This suggests that the large scale patterns of the differences between SMOS and Aquarius are due to differences in dielectric constant and their variation with SST. To quantify the impact of the dielectric constant model on the difference between SMOS and Aquarius, we compute the socalled error $E_{1}$ with respect to SMOS as

$$
E_{1}=\left|\mathrm{S}_{\mathrm{Aq}}-\mathrm{S}_{\mathrm{SMOS}}\right|-\left|S_{\mathrm{Aq} \_\mathrm{KS}}-S_{\mathrm{SMOS}}\right|
$$



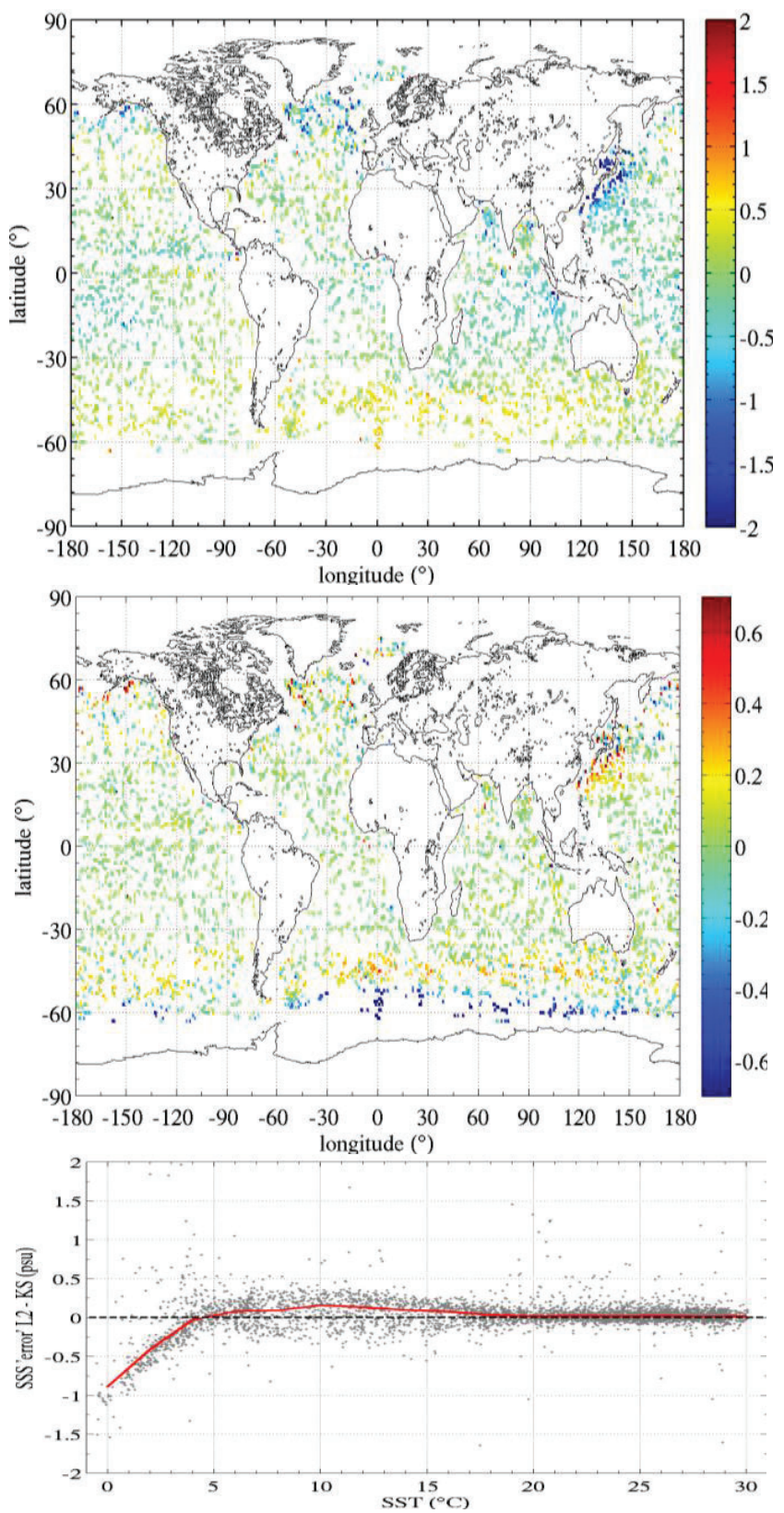

Fig. 5. (top) Map of SSS difference between Aquarius and Argo for Jan 2012. (middle) Map of the error difference with respect to Argo between Aquarius and Aquarius reprocessed using the KS model. (bottom) Data from (middle) are reported versus sea surface temperature. The red line is the median inside $2^{\circ} \mathrm{C}$ bins.

Positive $E_{1}$ values indicate that the SSS reprocessed with the KS model $\left(S_{\mathrm{Aq} \text { KS }}\right)$ are in better agreement with SMOS SSS $\left(S_{\mathrm{SMOS}}\right)$. Negative values show that the nominal Aquarius $\left(\mathrm{S}_{\mathrm{Aq}}\right)$ product is in better agreement with SMOS. As reported in Fig. 4 , using the KS model improves the agreement with SMOS in the $-45^{\circ}$ and $+45^{\circ}$ latitude bands by about $0.3 \mathrm{psu}$. The differences are much smaller and more mixed at lower latitudes. In the very high southern latitudes $\left(-60^{\circ}\right)$ and in coastal areas around North East Asia, south of Alaska and North East America, the results are more mixed (dark blue and red pixels close to each other). This suggest that the differences are due to various factors in addition to the dielectric constant model, for example the presence of sea ice (at latitudes higher than $-60^{\circ}$ ) or RFI in coastal areas. However, there is an overall degradation of the comparisons with SMOS when using the KS model for very cold waters (less than $3^{\circ} \mathrm{C}$ ).

\section{COMPARISON WITH In Situ ARGO DATA}

To determine whether the differences observed when using the KS model provide an improvement or a degradation of the Aquarius product, we compare the nominal Aquarius data and the reprocessed data to in situ measurements from the Argo network of drifting floats [13]. We first compute monthly average salinity maps from the Argo data at $1^{\circ}$ by $1^{\circ}$ spatial resolution in latitude and longitude, keeping all valid measurements performed at depth of $10 \mathrm{~m}$ or less. Then we compute the difference between these maps and those obtained from the Aquarius data with and without reprocessing.

The spatial distribution of the differences between the nominal Aquarius product and the Argo data (Fig. 5, top) shows similarities with Fig. 1 (top) and Fig. 3. Aquarius SSS is larger in the latitude bands $-45^{\circ}$ and $+45^{\circ}$. It is fresher in coastal areas in North East Asia, south of Alaska and in North East America. We do not observe the fresh value at the very high southern latitudes, although few Argo data are available there. For waters warmer than $25^{\circ} \mathrm{C}$, both the nominal and reprocessed Aquarius data, as well as SMOS data, show fresher SSS than the buoys (not shown).

To quantify the improvement or degradation due to the reprocessing of Aquarius data, we compute the error with respect to Argo similarly as in (1)

$$
E_{2}=\left|\mathrm{S}_{\mathrm{Aq}}-\mathrm{S}_{\mathrm{Argo}}\right|-\left|S_{\mathrm{Aq} \_\mathrm{KS}}-S_{\mathrm{Argo}}\right|
$$

As reported in Fig. 5 (middle), the reprocessed Aquarius data show improved agreement with Argo data in the latitude band around $-45^{\circ}$, and in a few coastal areas (e.g. coastal East Asia). Significant degradation is observed around $-60^{\circ}$ and for waters colder than $3^{\circ} \mathrm{C}$ (Fig. 5, bottom).

\section{DIFFERENCES IN ANCILLARY SSS PRODUCTS USED FOR VICARIOUS CALIBRATION}

The differences in the reference SSS field used for the calibration of the SMOS and Aquarius radiometers are most of the time relatively small, but not always negligible (Fig. 6, top). Large regions of the ocean exhibit differences of just 0.1 psu or less. However, regional differences can be significantly larger (1 psu or more) and are variable in time (not shown). An important region to assess is the one used for SMOS calibration, where the OTT is performed (red square in Fig. 6, top). The differences in the OTT region vary spatially by a few tenths of a psu. The average of the difference over the OTT region changes temporally between $-0.1 \mathrm{psu}$ and $+0.05 \mathrm{psu}$ since Aquarius started operating in 2011 (Fig. 6, bottom). This is a significant difference for a calibration target. It will be necessary to assess what the impact is on the final calibration of the instruments. 

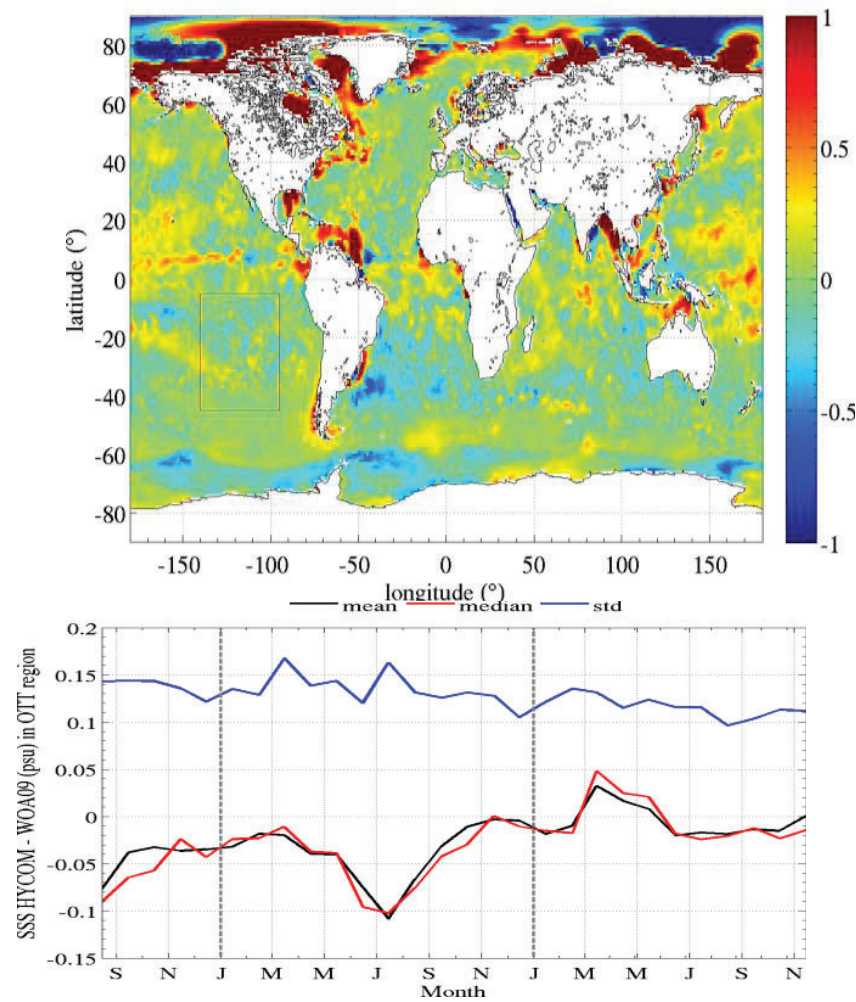

Fig. 6. (top) Global monthly map of the differences in SSS (psu) between the two ancillary products used in the calibration of Aquarius and SMOS. The difference is between the HYCOM model (used for Aquarius) and the World Ocean Atlas (used for SMOS). The red square in the south of the Pacific Ocean off the coast of South America illustrates the region used for the calibration of SMOS SSS product (i.e. the Ocean Target Transformation). (bottom) Time series of the average (mean and median) and standard deviation of the difference in SSS between HYCOM and WOA over the Ocean Target Transformation (OTT) region since the start of the Aquarius mission (Aug 2011 - Nov 2013). The vertical dashed lines part the different years.

\section{CONCLUSIONS AND DISCUSSION}

We assessed the difference in SSS retrieved by SMOS and Aquarius spaceborne radiometers. Both instruments observe similar large scale patterns, but also report significant regional discrepancies (mostly between $+/-1 \mathrm{psu}$ ). SMOS in particular exhibits significant freshening in coastal areas, likely due to contamination of the radiometric signal due to land. SMOS SSS are in general fresher, except at the very high southern latitudes near the ice edge and at a few local (mostly coastal) areas. The differences exhibit large scale pattern similar to SST variation.

To investigate the source of the observed differences, we reprocess the Aquarius SSS using the sea water dielectric constant model used for SMOS. The reprocessing includes the re-calibration of the Aquarius data. The results show improved comparisons between Aquarius and SMOS SSS by a few tenths of a psu for temperatures between $6^{\circ} \mathrm{C}$ and $18^{\circ} \mathrm{C}$. Warmer waters show little difference. Water colder than $3^{\circ} \mathrm{C}$ show mixed results, probably due to a complex mix of error sources (presence of sea ice, high surface roughness and currents).
The comparison of the reprocessed SSS with in situ data from the Argo network of drifting buoys shows improvement of a few tenths of a psu for temperatures between $6^{\circ} \mathrm{C}$ and $18^{\circ} \mathrm{C}$. For warmer waters, both the nominal and reprocessed Aquarius data, as well as SMOS data, show fresher SSS than the buoys. For very cold waters (less than $3^{\circ} \mathrm{C}$ ), the reprocessed data show significant degradation of the SSS compared to the buoys.

We also assessed the differences in the ancillary reference SSS fields used for the calibration of both instruments. We find that the differences could explain biases of up to $0.1 \mathrm{psu}$, varying in time.

The possible correlation between various sources of error is still being investigated. For example, temperature is often correlated with roughness (e.g. the cold waters at high southern latitudes also have high winds and strong currents), therefore it is important to indentify the actual source of the discrepancies. In addition, some differences have been identified between various SST ancillary products [14]. Such differences could explain part of the differences we have identified in our study. Similarly to what we have done here with SSS, the impact of differences in SST products is being evaluated.

\section{REFERENCES}

[1] Y. Kerr, et al., "The SMOS mission: New tool for monitoring key elements ofthe global water cycle," Proceedings of the IEEE, vol. 98, no. 5, pp. 666-687, May 2010.

[2] D. Le Vine, G. S. E. Lagerloef, and S. Torrusio, "Aquarius and remote sensing of sea surface salinity from space," Proceedings of the IEEE, vol. 98, no. 5, pp. 688-703, May 2010.

[3] E. P. Dinnat and D. M. Le Vine, "Impact of sun glint on salinity remote sensing: An example with the Aquarius radiometer," IEEE Trans. Geosci. Remote Sensing, vol. 46, no. 10, pp. 3137-3150, October 2008.

[4] D. M. Le Vine, E. P. Dinnat, , S. Abraham, P. de Matthaeis, and F. J. Wentz, "The Aquarius simulator and cold-sky calibration," IEEE Trans. Geosci. Remote Sensing, vol. 49, no. 9, pp. 3198-3210, September 2011.

[5] X. Yin, J. Boutin, and P. Spurgeon, "Biases between measured and simulated SMOS brightness temperatures over ocean: Influence of Sun," IEEE Journal of Selected Topics in Applied Earth Observations and Remote Sensing, vol. 6, no. 3, pp. 1341-1350, June 2013.

[6] E. P. Dinnat, J. Boutin, G. Caudal, J. Etcheto, and P. Waldteufel, "Influence of sea surface emissivity model parameters at L-band for the estimation of salinity," International Journal of Remote Sensing, vol. 23, no. 23, pp. 5117-5122, December 2002.

[7] E. P. Dinnat, J. Boutin, G. Caudal, and J. Etcheto, "Issues concerning the sea emissivity modeling at L-band for retrieving surface salinity," Radio Science, vol. 38, no. 4, pp. 25-1-25-11, May 2003.

[8] L. A. Klein and C. T. Swift, "An improved model for the dielectric constant of sea water at microwave frequencies," IEEE Trans. Antennas Propagat., vol. AP-25, no. 1, pp. 104-111, 1977.

[9] T. Meissner and F. Wentz, "The emissivity of the ocean surface between 6 and $90 \mathrm{GHz}$ over a large range of wind speeds and earth incidence angles," IEEE Trans. Geosci. Remote Sensing, vol. 50, no. 8, pp. 30043026, Aug 2012.

[10] J. Piepmeier, et al., "Aquarius radiometer post-launch calibration for product version 2," NASA, Tech. Rep. AQ-014-PS-0015, 2013.

[11] J. Antonov, et al., "World ocean atlas 2009 volume 2: Salinity," in NOAA Atlas NESDIS 69, S.Levitus, Ed. Washington, D.C., USA: U.S. Government Printing Office, p. 184, 2012.

[12] E. P. Chassignet et al., "The HYCOM (HYbrid Coordinate Ocean Model) data assimilative system," Journal of Marine Systems, vol. 65, Issues 1-4, pp. 60-83, March 2007.

[13] http://www.argo.net/ 
[14] X. Yin, J. Boutin, E. Dinnat, N. Martin, and S. Guimbard, "SMOS SSS uncertainties associated with errors on auxiliary parameters," in European Geosciences Union General Assembly, Vienna, Austria, Apr 27 - May 2, 2014 